\title{
Biotransformation of acetophenone to R-1-phenylethanol with immobilized Pichia capsulata in batch reactor
}

\author{
Viera Illeová, Pavel Ačai, Jakub Féher, Milan Polakovič \\ Institute of Chemical and Environmental Engineering, \\ Slovak University of Technology, Bratislava \\ viera.illeova@stuba.sk
}

\begin{abstract}
Biotransformation of acetophenone to R-1-phenylethanol with Pichia capsulata immobilized in Caalginate gel beads was studied. Experiments were carried out in a batch reactor stirred on an orbital shaker $(300 \mathrm{rpm})$ at $25^{\circ} \mathrm{C}$. Based on experimental data, uncoupled parameters of reaction kinetics (measurements with free cells) and diffusion coefficients (sorption method) were estimated. The model expressing the conversion of acetophenone with immobilized cells in the batch reactor consisted of mass balance equations of acetophenone and R-1-phenylethanol in the liquid and solid phases. A satisfactory agreement between the experimental and predicted values of acetophenone and R-1-phenylethanol concentrations was achieved
\end{abstract}

Key words: immobilization, Pichia capsulata chiral alcohols, effective diffusion coefficient, kinetic parameters, reaction-diffusion model prediction

\section{Introduction}

Chiral alcohols are one of the most useful groups of chiral compounds. They are readily obtained by hydrogenation of ketones with Rh- and Ru-diphosphine catalysts on a large scale, (Blaser 2003), but isolated enzymes catalyse enantioselective reduction with remarkably better chemo-, regio-, and stereoselectivity. Furthermore, bioreduction is usually possible under mild reaction conditions. Enantioselective bioreduction of aromatic ketones requires the presence of enzymes - alcoholdehydrogenases (ADHs), exhibiting catalytic activity only in the presence of a cofactor (mostly NADPH or NADH). Rarely, these enzyme-bound cofactors are from the group of flavines (FAD) or pyrroloquinoline quinone (PQQ) (Walsh, 1980). However, all mentioned cofactors are too expensive to be used stoichiometrically - with prices up to 2,700,000 € per mol of PQQ (Leonida, 2001). Another disadvantage is the stability of the cofactor, wherein NADH is much more stable under the operational conditions than NADPH. In comparison to isolated enzymes, whole cells have internal cofactor regeneration, and thus an addition of cheap glucose or ethanol is sufficient to drive the reaction. In addition, whole cells are easier and cheaper to obtain than pure enzyme.

Asymmetric reduction of acetophenone to the corresponding secondary alcohols ( $\mathrm{R}, \mathrm{S}-\mathrm{l}-$ phenylethanol) with relatively good conversion and selectivity has been extensively studied by many authors. However, overall productivity of homochiral alcohols including the isolation procedure is still relatively low and therefore more expensive than the conventional (chemical) processes. Different feeding strategies reducing the substrate toxicity (Valadez-Blanco et al., 2009; Cheng, 1996), treatment of the resting cell with acetone (Matsuda et al., 2000) or immobilization have been used to improve the productivity and enantiomeric excess. One of the reports (Hasegawa et al., 1998) on acetophenone bioreduction introduces Geotrichum candidum immobilized onto an ion exchange resin with polyallylamine. Regulated temperature, $\mathrm{pH}$ and a suitable buffer contributed to up to $99 \%$ enantiomeric excess (e.e) of S-l-phenylethanol. In addition, stability of the catalyst was enhanced so that it could be recycled for four times without any significant loss in the reaction yield. Hasegawa (Hasegawa et al., 1998) performed the biotransformation of acetophenone using Hansenula capsulata cells in a calcium-alginate gel. They showed that the productivity of S-l-phenylethanol was better in a packed bed than in a batch reactor because of its anaerobic conditions and the prevented reverse oxidation. Kurbanoglu investigated repeated use of Rhodotorula glutinis cells in a batch reactor with $1 \mathrm{mmol} \cdot \mathrm{l}^{-1}$ of acetophenone (Kurbanoglu et al., 2010). This strain also produced S-l-phenylethanol and the total yield was $70 \%$ even after 15 repeated cycles. In view of the above facts, it seems that most of these biocatalysts follow the Prelog's rule (Prelog 1964). Anti-Prelog R-specific whole cell biocatalysts are rarer (Hummel, 1990; Schlieben et al., 2005; Protsko et al., 2010) and all except one require NADPH as a cofactor. Leifsonia species was found to be the sole source of NADH-dependent enzyme producing the R-form of 1-phenylethanol (Inoue et 
al. 2005). Hence, a combination of the $\mathrm{R}$ preference and NADH are more desired due to more efficient regeneration and lower price of the cofactor. It is well known that the screening of a wide variety of microorganisms is an efficient method to obtain catalyst with required properties. Therefore, the objective of our previous work was to find strains that are able to produce $\mathrm{R}$ 1-phenylethanol with excellent enantiomeric purity and high yield; with NADH dependence as a surprising bonus. Among the 23 wild yeasts strains tested, only Pichia capsulata ATCG 16753 meets both required criteria. Concentration of glucose, control of oxygen during cultivation as well as cultivation time contribute to high yield and high enantioselectivity of the mentioned homochiral 1-phenyethanol (Homola et al., 2015). As discussed in the introduction, immobilization of different S-specific microorganisms into hydrogels improved the enantiomeric excess, yield and stability of the catalyst in the cells. These results encouraged experiments with the same bioreduction process based on R-specific Pichia capsulata immobilized in calcium alginate gel.

In the present study, bioreduction of acetophenone by Ca-alginate immobilized Pichia capsulata was performed in a batch system. The reversible Michaelis-Menten equation was used to describe the bioreduction kinetics of free cells. Effective diffusion coefficients of acetophenone were determined by the sorption method with inactive biomass. Kinetic parameters determined from the experiment with free cells and effective diffusion coefficients were used to predict acetophenone and $\mathrm{R}$ 1-phenylethanol progress curves in the liquid phase during the process.

\section{Mathematical model}

\section{Determination of kinetics parameters}

Reaction kinetics with free cells in a batch stirred bioreactor was described using a model consisting of two ordinary differential equations with the following initial conditions:

$$
\begin{gathered}
\frac{d c_{A C F}}{d t}=\frac{k_{1} c_{c a t} c_{A C F}}{K_{M 1}+c_{A C F}}-\frac{k_{2} c_{c a t} c_{R-P H E}}{K_{M 2}+c_{R-P H E}} \\
\frac{d c_{R-P H E}}{d t}=-\frac{k_{1} c_{c a t} c_{A C F}}{K_{M 1}+c_{A C F}}+\frac{k_{2} c_{c a t} c_{R-P H E}}{K_{M 2}+c_{R-P H E}} \\
t=0, c_{A C F}=c_{A C F, 0}, c_{R-P H E}=c_{R-P H E, 0}=0
\end{gathered}
$$

where $c_{A C F}$ is the acetophenone (ACF) concentration, $c_{R-P H E}$ is the R-1-phenylethanol concentra- tion, $t$ is time, $k_{1}$ is the the kinetic parameter of the forward reaction, $k_{2}$ is kinetic parameter of the backward oxidation, $c_{c a t}$ is the biocatalyst concentration, $K_{M 1}$ and $K_{M 2}$ are Michaelis-Menten equation parameters for the forward and backward reactions, respectively, $c_{A C F, 0}$ is the initial acetophenone concentration, $c_{R \cdot P H E, 0}$ is the initial R-1-phenylethanol concentration.

Kinetic parameters were estimated by fitting the progress curve data using optimization procedures of the AthenaVisual Workbench software (Stewart \& Associates Engineering Software, Madison, WI, USA; http://www.athenavisual.com).

\section{Effective diffusion coefficients evaluation}

Values of effective diffusion coefficients of acetophenone and R-1-phenylethanol were determined by the sorption method with an immobilized inactive gel bead suspension of cells in a batch stirred vessel. The volume change of the liquid phase was also taken into account. The differential mass balance of components in the liquid and solid phases with initial and boundary conditions can be given by the following partial differential equations:

Liquid phase:

$$
\begin{gathered}
V_{L} \frac{\partial c_{b, i}}{\partial t}+c_{b, i} \frac{\partial V_{L}}{\partial t}= \\
=\left[\frac{(1-\varepsilon) \varepsilon_{p}}{\varepsilon} \frac{3}{R_{p}} D_{e, i} \frac{\partial c_{p, i}}{\partial r} /_{r=R_{p}}\right] V_{L} \\
t=0, c_{b, i}=c_{b 0, i}, V_{L}=V_{L 0}
\end{gathered}
$$

Solid phase:

$$
\begin{aligned}
\varepsilon_{p} \frac{\partial c_{p, i}}{\partial t} & =D_{e, i}\left(\frac{\partial^{2} c_{p, i}}{\partial r^{2}}+\frac{2}{r} \frac{\partial c_{p, i}}{\partial r}\right) \\
t & =0, c_{P, i}=0
\end{aligned}
$$

$$
\begin{aligned}
& r=0, \frac{\partial c_{p, i}}{\partial r}=0 \\
& r=R_{P}, c_{p, i}=c_{b, i}
\end{aligned}
$$

where $V_{L}$ is the liquid volume, $t$ is time, $c_{b, i}$ is the concentration of the species outside the particles in the fluid phase, $i$ is the considered component, $\varepsilon$ is the bed voidage, $\varepsilon_{p}$ is the particle porosity, $D_{e i}$ is the effective diffusion coefficient of acetophenone and R-1-phenylethanol, $R_{p}$ is the average particle radius, $\mathrm{r}$ is the distance from the particle centre, $c_{p, i}$ 
is the concentration of the species on the surface/ inside the particles in the solid phase, $c_{b 0, i}$ is the initial acetophenone concentration, and $V_{L O}$ is the initial volume of the liquid phase. It is assumed that values of $\varepsilon, \varepsilon_{p}$ and $R_{p}$ are constant.

A commercial process engineering software, AthenaVisual Workbench (Stewart \& Associates Engineering Software, Madison, USA; http:// www.athenavisual.com), was used for the effective diffusion coefficients estimation.

\section{Prediction of acetophenone and 1-phenylethanol in a batch stirred bioreactor with immobilized cells}

The proposed mathematical model of enantioselective biotransformation of acetophenone to R-1-phenylethanol with immobilized cells entrapped in Ca-alginate gel beads, in a batch stirred bioreactor, was based on the general rate model consisting of the mass balance equations of the considered species in the liquid and solid phases. The volume change of the liquid phase was also taken into account.

The differential mass balance of components in the liquid phase under initial conditions can be expressed as follows:

$$
\begin{gathered}
V_{L} \frac{\partial c_{b, i}}{\partial t}+c_{b, i} \frac{\partial V_{L}}{\partial t}= \\
=\left[\frac{(1-\varepsilon) \varepsilon_{p}}{\varepsilon} \frac{3}{R_{p}} D_{e, i} \frac{\partial c_{p, i}}{\partial r} /_{r=R_{p}}\right] V_{L} \\
t=0, c_{b, i}=c_{b 0, i}
\end{gathered}
$$

The differential mass balance of species for a particle under initial and boundary conditions is:

$$
\begin{gathered}
\varepsilon_{p} \frac{\partial c_{p, i}}{\partial t}=D_{e, i}\left(\frac{\partial^{2} c_{p, i}}{\partial r^{2}}+\frac{2}{r} \frac{\partial c_{p, i}}{\partial r}\right) \pm r_{i} \\
t=0, c_{P, i}=0 \\
r=0, \frac{\partial c_{p, i}}{\partial r}=0 \\
r=R_{P}, c_{p, i}=c_{b, i}
\end{gathered}
$$

where $r_{i}$ is the reaction term of acetophenone reduction.

Reaction rate expressions for reversible biotransformation of acetophenone to R-1-phenylethanol are given as:

$$
r_{A C F}=\frac{k_{1} c_{c a t} c_{p, A C F}}{K_{M_{A C F}}+c_{p, A C F}}-\frac{k_{2} c_{c a t} c_{p, R-P H E}}{K_{M_{P H E}}+c_{p, R-P H E}}
$$

$$
r_{R-P H E}=-\frac{k_{1} c_{c a t} c_{p, A C F}}{K_{M_{A C F}}+c_{p, A C F}}+\frac{k_{2} c_{c a t} c_{p, R-P H E}}{K_{M_{P H E}}+c_{p, R-P H E}}
$$

where $c_{p, A C E}$ and $c_{p, R-P H E}$ are concentrations of acetophenone and R-1-phenylethanol in the solid phase, respectively. The biocatalyst concentration in the solid phase, $c_{\text {cat }}$, is approximately 5.7 times higher than with free cells. It follows from the fact that progress curves for free and immobilized cells were performed with the same cells amount but with different volumes. Concentration profiles of acetophenone and R-1-phenylethanol in the liquid phase were predicted on the basis of kinetics parameters obtained for free cells and diffusion coefficients determined by sorption method with an immobilized inactive cell.

\section{Materials and methods}

\section{Chemicals}

Acetophenone, S-1-phenylethanol, R-1-phenylethanol, 1-phenylethanol, $\mathrm{NaOH}, \mathrm{H}_{2} \mathrm{SO}_{4}, \mathrm{HCl}, \mathrm{CaCl}_{2}$, $\mathrm{Na}_{2} \mathrm{SO}_{4}$, glucose, glycerol and ethyl acetate were obtained from Mikrochem (Slovakia). Bacteriological peptone and yeast extract were purchased from Oxoid (England).

\section{Cultivation}

Cells of Pichia capsulata ATCG 16753 were frozen in a solution of YPD (yeast, peptone, dextrose) medium at $-80{ }^{\circ} \mathrm{C}$. To avoid damage to the cells during freezing, glycerol in a concentration of $40 \%\left(\mathrm{w} \cdot \mathrm{vol}^{-1}\right)$ was added as a cryoprotectant to the medium.

YPD medium containing $2 \%\left(\mathrm{w} \cdot \mathrm{vol}^{-1}\right)$ of glucose, $2 \%\left(\mathrm{w} \cdot \mathrm{vol}^{-1}\right)$ of bacteriological peptone and $1 \%$ $\left(\mathrm{w} \cdot \mathrm{vol}^{-1}\right)$ of yeast extract was used for storage and inoculation steps. Inoculation medium contained only $1 \%\left(\mathrm{w} \cdot \mathrm{vol}^{-1}\right)$ of glucose. Initial $\mathrm{pH}$ of the media was adjusted to 5.8 with $\mathrm{H}_{2} \mathrm{SO}_{4}$ and the cells were grown at their temperature optima $\left(24^{\circ} \mathrm{C}\right)$ (Yamada 1994). The volume of $15 \mathrm{ml}$ of the stock culture solution was inoculated into $150 \mathrm{ml}$ of sterile YPD media in a $500 \mathrm{ml}$ Erlenmayer flask and the cell were grown in a shaking incubator (New Brunswick, England) at $250 \mathrm{rpm}$. After $18 \mathrm{~h}$ of cultivation, $450 \mathrm{ml}$ of inoculum were aseptically transferred into $3.15 \mathrm{l}$ of media. Biomass was produced in a 41 laboratory fermenter equipped with a control unit (Sartorius, Germany) at constant $\mathrm{pH}$ (5.8) adjusted with $\mathrm{NaOH}$ 
and $\mathrm{H}_{2} \mathrm{SO}_{4}$. Stirring at the beginning of cultivation was set to $300 \mathrm{rpm}$ and the oxygen was maintained at $5 \%$ of saturation during the whole cultivation by mixing nitrogen and air and by increasing the stirrer speed gradually. Cultivation was finished after 15 hours when the concentration of ethanol was close to zero.

\section{Processing of samples and final biomass from cultivation}

During fermentation, $5 \mathrm{ml}$ of samples were taken every 1.5 hour. Concentration of ethanol in supernatant was measured using HPLC (Agilent, USA) equipped with a refractive index detector (Homola 2015). At the end of cultivation, the biomass was collected by centrifugation (Hettich, Germany) ( $\mathrm{T}=6{ }^{\circ} \mathrm{C}, 9000 \mathrm{rpm}$ for $10 \mathrm{~min}$ ), three times washed with water and mixed with a buffer $\left(0.1 \mathrm{~mol} \cdot \mathrm{l}^{-1}\right.$ phosphate, $\left.\mathrm{pH} 5\right)$ in the exact mass ratio of $1: 3$ on an analytical balance. The amount of dry biomass in suspension was determined using a moisture analyzer (Kern, Germany). 17.1 \% of dry biomass in suspension was determined as the mean of three replicates.

\section{Biomass immobilization}

Immobilization procedure of inactivated cells was the same as that of active cells. In both cases, the cells suspension (17.1\% of dry biomass) was mixed with $3 \%\left(\mathrm{w} \cdot \mathrm{vol}^{-1}\right)$ of a water alginate solution in the exact mass ratio of $1.2: 1$ on an analytical balance. Then, the homogenized solution was placed into a syringe and dropped through it into a $0.2 \mathrm{~mol} \cdot \mathrm{l}^{-1} \mathrm{CaCl}_{2}$ solution. The distance between the nozzle and the calcium bath was $8 \mathrm{~cm}$. Coaxial air-flow technology was used for droplet generation. In order to obtain the desired size of spherical particles in reasonable time, the nozzle size varied from $8 \mathrm{~mm}$ to $12 \mathrm{~mm}$. Three sets of particles with different diameters were prepared by a combination of air flow and the needle size. Particles were stirred at the laboratory temperature for at least $1.5 \mathrm{~h}$ to complete gel formation and then decanted and washed with redistilled water. Diameter of the particles was measured with an Abbe comparator (Carl Zeiss, Germany). The prepared beads had a narrow size distribution with a relative standard deviation of up to $6 \%$. The mean diameter for each set of particles was calculated from the diameters of 50 randomly chosen particles.

\section{Determination of diffusion coefficient}

To uncouple the diffusion-reaction process, it was necessary to measure the diffusion coefficient with inactive biomass. Therefore, the cells were inactivated in the first step with $\mathrm{HCl}$ by the procedure of $\mathrm{Wu}(\mathrm{Wu}, 1994)$ and immobilized using the procedure mentioned above. Then, the diffusion coefficient was measured at $25{ }^{\circ} \mathrm{C}$ in a batch reactor. Total volume of beads (solid fraction, $V_{S}$ ) and the acetophenone solution (liquid fraction, $\left.V_{L}\right)$ was $50 \mathrm{ml}$. The ratio between $V_{S}$ and $V_{L}$ was $1: 5$. Since preliminary experiments had shown rapid diffusion of acetophenone into particles, in the first three minutes, the samples were taken at the shortest possible intervals. Steady state was achieved for all particle diameters in about $15 \mathrm{mi}$ nutes. Concentration of acetophenone in bulk was determined using HPLG with an RI detector based on the calibration line. Effective diffusion coefficients for particles with different diameters were determined by one parameter optimization from equations (4-8).

\section{Reaction experiments}

Progress curves for free cells and immobilized particles were performed with the same amount of cells, but with different reaction volume. Therefore concentration of biocatalyst in heterogeneous system was increased by factor 5.7. The reaction was performed so that the amount of dry biomass in the reaction medium was $36 \mathrm{~g} \cdot \mathrm{l}^{-1}$, initial concentration of acetophenone was $1 \mathrm{~g} \cdot \mathrm{l}^{-1}$ and that of glucose was $50 \mathrm{~g} \cdot \mathrm{l}^{-1}$. The reaction was initiated by an addition of the proper amount of acetophenone and glucose in $0.1 \mathrm{~mol} \cdot \mathrm{l}^{-1}$ phosphate buffer with $\mathrm{pH} 5$ into a $50 \mathrm{ml}$ Erlenmayer flask with cell suspension in the same phosphate buffer. The flask with the reaction medium was stirred on an orbital shaker (300 rpm) at $25^{\circ} \mathrm{C}$. Overall reaction time was 6 hours. During the reaction, $0.8 \mathrm{ml}$ of samples at appropriate time intervals was directly mixed with the same volume of ethyl acetone (extraction solvent) in order to stop the reaction in the samples. The mixture of the sample and the extraction solvent was stirred for 10 minutes on a vortex and then centrifuged $\left(\mathrm{T}=6{ }^{\circ} \mathrm{C}, 9000 \mathrm{rpm}\right.$ for $10 \mathrm{~min}$ ). The upper organic phase was removed with a pipette and mixed with $\mathrm{Na}_{2} \mathrm{SO}_{3}$ to remove water.

Then, the samples were filtered into vials and the concentrations of the acetophenone, R-1-phenylethanol and S-1-phenylethanol were determined using GC (Agilent Technologies, USA) under conditions previously published (Homola, 2015). The enantiomeric excess (e.e) was calculated from known concentrations of each enantiomers using the equation:

$$
e . e=\frac{c_{R-P H E}-c_{S-P H E}}{c_{R-P H E}+c_{S-P H E}} \cdot 100 \%
$$


where $c_{R \cdot P H E}, c_{S-P H E}$ are R-1-phenylethanol and R-1phenylethanol concentrations, respectively.

\section{Result and discussion}

The first key step of our experimental work was to produce biomass with the highest possible specific activity with respect to the $R$-form. Initial glucose concentration of $10 \mathrm{~g} \cdot \mathrm{l}^{-1}$ and controlled oxygen concentration (5\% saturation) were chosen based on previous laboratory results, where cultivation conditions were shown to influence the biocatalyst properties. Based on monitoring of glucose and ethanol concentration, cultivation was finished after 15 hours when the ethanol concentration was close to zero and biomass reached the highest selectivity.

Knowledge of the diffusion coefficient is necessary for correct evaluation of kinetic parameters in heterogeneous catalysis. However, to uncouple the diffusion-reaction process, it was necessary to measure the diffusion coefficient with inactive biomass. Therefore, the cells were first inactivated with $\mathrm{HCl}$, and then immobilized. Effectiveness of cell inactivation was confirmed by a simple batch experiment. The results indicate no conversion of acetophenone by the cells during the whole and relatively long experiment (25 hours) (data not shown).

Immobilization of active and inactive biomass was carried out employing the same procedure and under the conditions described in Material and methods. In preliminary experiments, the influence of various diameters of the nozzles and air flow on the particle diameter and sphericity was investigated. By combination of corresponding needle and air-flow, three different diameters $(1.17,1.69,2.34 \mathrm{~mm})$ of Ca-alginate gel beads were obtained. When spherical beads were suspended in an appropriate acetophenone solution with an initial concentration of $\mathrm{c}_{\mathrm{L} 0}$, the substrate diffused into the beads and its concentration just on the surface of a bead equals the concentration in the liquid phase. Acetophenone concentration in the solution decreased rapidly just after the addition of the beads, and gradually slowed down with time, leveling off after about 15 min when the equilibrium was reached. For each diameter, three experiments were performed at the same conditions and the mean value of effective diffusion coefficient was calculated. Measurement of the diffusion coefficient for one size particles $(\mathrm{d}=2.34 \mathrm{~mm})$ is presented in Fig. 1. A relative good fit of experimental data was achieved. The effective diffusion coefficients of acetophenone for particles with different diameters were calculated from equation 6 and are summarized in
Table 1. The results reviewed in this table point out the correlation between the values of effective diffusion coefficients and the particle diameters. Differences in the pore structure of particles occurring during the immobilization (Dursun et al., 2005) could be the possible explanation of the effective diffusion coefficients variations. For the prediction of acetophenone reduction to 1-phenylethanol with immobilized cells, the effective diffusion coefficients of R-1-phenylethanol were approximated by the values of acetophenone effective diffusion coefficients.

Kinetic experiments of acetophenone reduction to R-1-phenylethanol by a Pichia capsulata whole-cell biocatalyst $\left(36 \mathrm{~g} \cdot \mathrm{l}^{-1}\right)$ were carried out at optimal $\mathrm{pH}$ (pH 5), temperature of $25^{\circ} \mathrm{C}$ and glucose concentration of $50 \mathrm{~g} \cdot \mathrm{l}^{-1}$ (Homola et al., 2015). The progress curves of acetophenone and R-1-phenylethanol were applied to collect the kinetic data. This reduction showed a reversible character, apparent equilibrium was reached after about 6 hours. At the end of the reaction, conversion of the substrate was $60 \%$ and the enantiomeric excess was $56 \%$. Glucose concentration of $50 \mathrm{~g} \cdot \mathrm{l}^{-1}$ was sufficient to regenerate $\mathrm{NADH}$ and therefore acetophenone is the only limiting substrate. Based on the above, a model was developed to fit the experimental data considering the reversible Michaelis-Menten equation (Eq. 1-3). The quality of the fit was confirmed also by Fig. 2. A good match of the experimental and modeled values of the acetophenone and R-1phenylethanol concentrations was achieved.

Results of the parameter estimation are presented in Table 2. Both progress curves were satisfactorily smoothed with the model as confirmed by the value of the sum square of residual $\left(7.55 \times 10^{-3}\right)$. Kinetic

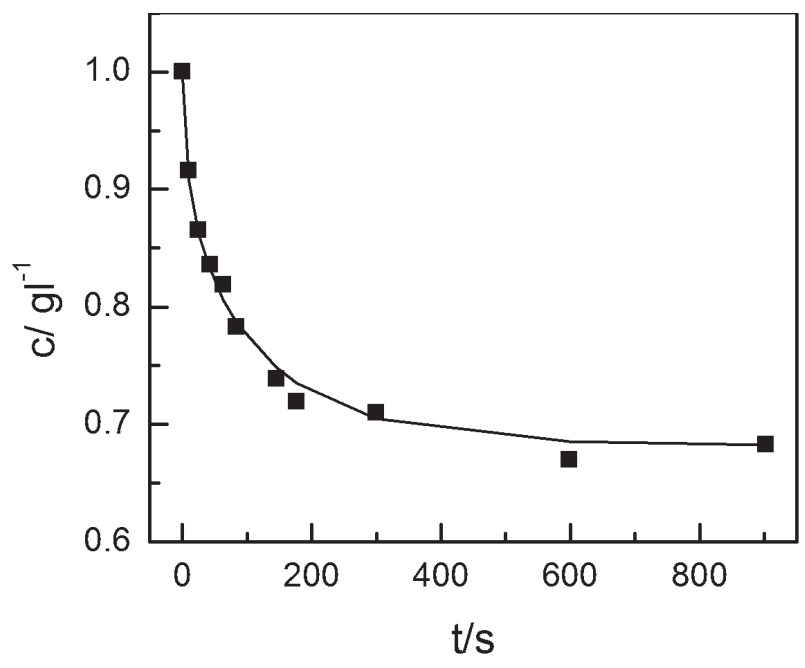

Fig. 1. Diffusion of acetophenone into alginate particles with inactive cells.

Line represents calculated concentration profile. 
Tab. 1. Dependence of effective diffusion coefficient on particle diameter.

\begin{tabular}{ccc}
\hline * $\mathbf{d} / \mathbf{c m}$ & ${ }^{* * *} \boldsymbol{D} \boldsymbol{e}_{\boldsymbol{i}} / \boldsymbol{m}^{2} \boldsymbol{s}^{-1}$ & $\begin{array}{c}\mathbf{9 5} \% \text { confidence } \\
\text { interval }\end{array}$ \\
\hline$(1.17 \pm 0.17) \times 10^{-1}$ & $2.44 \times 10^{-10}$ & $\pm 0.36 \times 10^{-10}$ \\
$(1.69 \pm 0.28) \times 10^{-1}$ & $3.61 \times 10^{-10}$ & $\pm 0.37 \times 10^{-10}$ \\
$(2.34 \pm 0.55) \times 10^{-1}$ & $6.17 \times 10^{-10}$ & $\pm 0.51 \times 10^{-10}$ \\
\hline
\end{tabular}

${ }^{*}$ Mean diameters of alginate particles with standard deviation. ${ }^{* *}$ Effective diffusion coefficients with $95 \%$ confidence interval.

parameters of both, the forward and the backward, reactions listed in Table 2 were estimated with a sufficient accuracy. Table 2 further shows that the Michaelis constant for the forward reaction was significantly higher than that of the backward reaction, indicating stronger affinity of the enzyme towards the substrate. On the other hand, negligible influence of the backward reaction is manifested by the relatively small difference between the rate constants. Rate constant $k_{2}$ is only 1.6 times lover than $k_{1}$. It means that the backward reaction contributes significantly to the relatively low substrate conversion. In our previous publication it was shown that low glucose concentration may be one of the reasons of the reaction in the opposite direction; from R-1-phenylethanol to acetophenone. The second reason for the increase of the backward reaction impact is the presence of oxygen. Oxidation of 1phenylethanol requires atmospheric oxygen; therefore, Hasegawa filled the reactor headspace with an inert gas (Hasegawa et al., 1998). No decrease in the concentration of 1-phenylethanol was observed in anaerobic conditions.

Tab. 2. Kinetic parameters of acetophenone reduction by free whole-cell biocatalyst at $\mathrm{pH} 5$ and temperature of $25^{\circ} \mathrm{C}$.

\begin{tabular}{lcc}
\hline Parameter & Value & $\begin{array}{c}\text { 95 \% *confidence } \\
\text { interval }\end{array}$ \\
\hline$k_{1} / \mathrm{g} \cdot \mathrm{min}^{-1} \cdot \mathrm{g}^{-1}$ & $3.1 \times 10^{-4}$ & $\pm 0.72 \times 10^{-4}$ \\
$K_{M 1} / \mathrm{g} \cdot \mathrm{l}^{-1}$ & $9.51 \times 10^{-1}$ & $\pm 3.33 \times 10^{-1}$ \\
$k_{2} / \mathrm{g} \cdot \mathrm{min}^{-1} \cdot \mathrm{g}^{-1}$ & $1.88 \times 10^{-4}$ & $\pm 0.11 \times 10^{-4}$ \\
$K_{M 2} / \mathrm{g} \cdot \mathrm{l}^{-1}$ & $1.49 \times 10^{-1}$ & $\pm 0.44 \times 10^{-1}$ \\
\hline
\end{tabular}

${ }^{*} 95 \%$ confidence interval of calculated kinetics parameters.

In the following experimental work, bioreduction reactions were performed using three sizes of alginate particles. Concentrations of acetophenone and biomass in the particles were comparable to the homogenous reaction. Results are summarized in Table 3. In case of the heterogeneous reaction, final conversion was higher for all three sets of alginate particles in comparison with free cells. Also

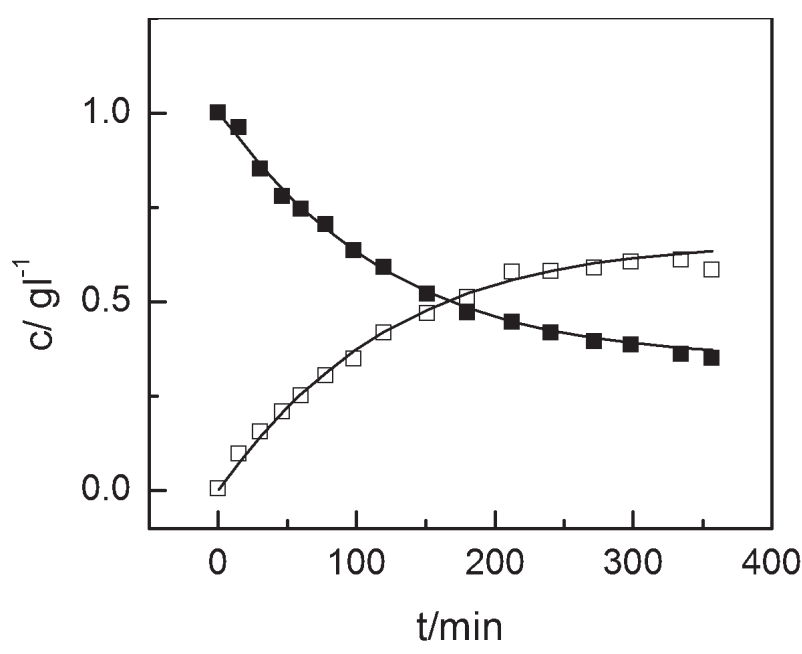

Fig. 2. Progress curves of acetophenone ( and R-1-phenyelthanol ( $\square$ ) for free cells.

Lines represent data fits from a model of reversible Michaelis-Menten equation.

$\mathrm{R}$ preference was higher, lower concentration of S-1-phenyethanol was observed (data not shown). Matsuda observed that enantiomeric excess of asymmetric bioreduction of acetophenone by Geotrichum candidum increased from $90 \%$ up to $99 \%$ when Geotricum candidum was immobilized (Matsuda et al., 2008). Nakamura studied asymmetric bioreduction by immobilized baker's yeast and observed significant change in e.e comparison to free cells of baker's yeast. He proposed that stereoselectivity could be controlled by immobilization (Nakamura et al., 1990). Physiological state of the cells and the regime of cultivation are also important factors affecting the stereochemical course of the reaction (Willaert et al., 1996).

Values of kinetic parameters and effective diffusion coefficients were indispensable for the solution of the reaction-diffusion model of acetophenone reduction to R-1-phenylethanol in a batch bioreactor with immobilized cells. Concentration profiles of acetophenone and R-1-phenylethanol in this heterogeneous system were predicted by simulations using equations $(9-15)$. The experimental and predicted values of acetophenone and R-1-phenylethanol concentrations show identical trends but some deviations were observed (Fig. $3 a-3 c)$. Calculated values of the effectiveness factors were about 0.94 (results are not shown). It can be supposed that the reaction was very little limited by diffusion. Considering the parameters used to describe the kinetics of immobilized cells, the match is very good. It means that the immobilization did not change the kinetic properties significantly compared to free cells. No significant loss of active cells occurred either. 

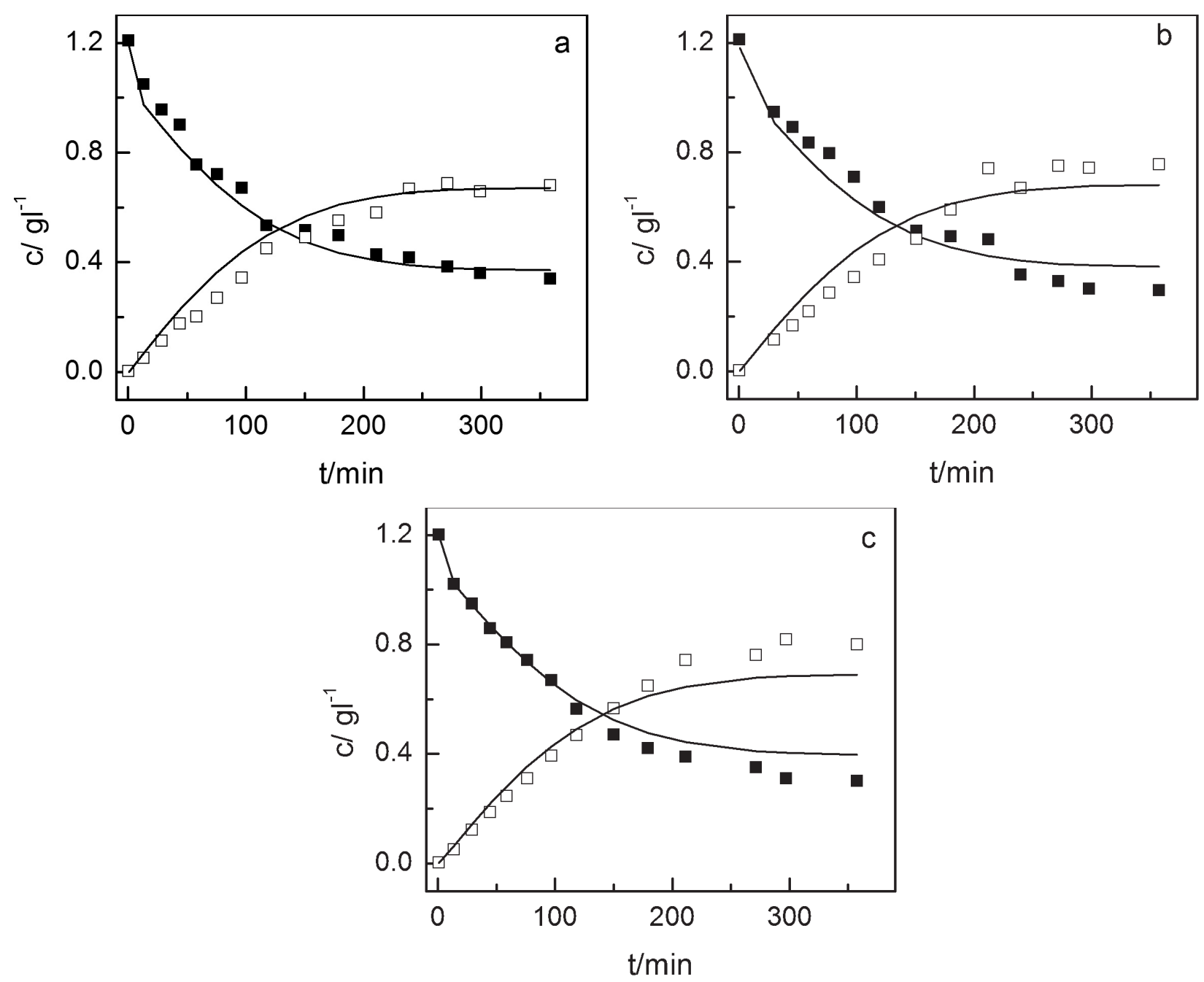

Fig. 3a-3c. Progress curves of acetophenone $(\square)$ and R-1-phenyelthanol ( $\square$ ) for particles with different diameter: $\mathrm{a}-1.17, \mathrm{~b}-1.69, \mathrm{c}-2.34 \mathrm{~mm}$. Lines represent the simulated concentration courses obtained from the reaction-diffusion model using diffusion coefficients from Table 1 and kinetic parameters from Table 2.

Tab. 3. Conversion of acetophenone and enantiomeric excess in reaction with free and immobilized cells.

\begin{tabular}{ccc}
\hline${ }^{* * *} \mathbf{d} / \mathbf{c m}$ & conversion/\% & e.e/\% \\
\hline free cells & 60.6 & 56.7 \\
\hline$(1.17 \pm 0.17) \times 10^{-1}$ & 72.2 & 64.8 \\
$(1.69 \pm 0.28) \times 10^{-1}$ & 75.7 & 67.6 \\
$(2.34 \pm 0.55) \times 10^{-1}$ & 75.2 & 67.5 \\
\hline
\end{tabular}

\section{Conclusion}

Biotransformation of acetophenone to R-1-phenylethanol was carried out in a well-stirred batch bioreactor with immobilized cells of Pichia capsulata entrapped in Ca-alginate gel beads. The analysis of this complicated biochemical process in a heterogeneous system was based on the identification of individual effects (internal diffusion, reaction) occurring simultaneously. The non-investigated effect was eliminated either by the minimization of its influence or by its total elimination. Kinetic parameters of enzyme reactions were estimated by non-linear regression analysis from the differential data obtained for free cells. Values of the effective diffusion coefficients were determined by the sorption method using immobilized inactive gel bead suspension of Pichia capsulata. Evaluated kinetic parameters and effective diffusion coefficients were used in the reaction-diffusion model containing partial differential equations for the prediction of acetophenone and 1-phenylethanol progress curves in the liquid phase during the process. A good match was obtained between the simulated and experimental data.

\section{Acknowledgement}

This article was created with the support of the Ministry of Education, Science, Research and Sport of the Slovak 
Republic within the Research and Development Operational Programme for the project "University Science Park of STU Bratislava”, ITMS 26240220084, co-funded by the European Regional Development Fund.

\section{Abbreviation}

ADHs alcoholdehydrogenases

PQQ pyrroloquinoline quinone

ACF acetophenone

PHE phenylethanol

YPD yeast, peptone, dextrose medium

e.e enantiomeric excess

\section{References}

Blaser HU, Malan C, Pugin B, Spindler F, Steiner H, Studer M (2003) Advanced Synthesis and Catalysis 345: 103-151.

Cheng C, Ma JH (1996) Process Biochemistry 31: 119-124.

Dursun AY, Tepe O (2005) Journal of Hazardous Materials B 126: 205-111.

Hasegawa Y, Adachi S, Matsuno R (1998) Journal of Fermentation and Bioengineering 85: 322-327.

Homola P, Kurák T, Illeová V, Polakovič M (2015) Food and bioproduct processing 96: 126-132.

Hummel W (1990) Biotechnology Letters 12: 403-408.
Inoue K, Makino Y, Itoh N (2005) Applied Environmental Microbiology 71: 3633-3641.

Kurbanoglu EB, Zilbeyas K (2010) Tetrahedron: Asymmetry 21: 461-464.

Leonida MD (2001) Current Medical Chemistry 8: 345-369.

Matsuda T, Marukado R, Mukouyama M (2008) Tetrahedron: Asymmetry 19: 2272-2275.

Matsuda T, Harada T, Nakajima N, Nakamura K (2000) Tetrahedron Letters 41: 4135-4138.

Nakamura K, Kawai Y, Nakajima N, Ohno A (1990) Journal of Organic Chemistry 56: 4778-4783.

Prelog V (1964) Pure Applied Chemistry 9: 119-130.

Protsko C, Vieille V, Laivenieks M, Prasad L, Sanders DAR, Delbaere LTL (2010) Acta Crystallographica Section F: Structural Biology and Crystallization Communications 66: 831-833.

Schlieben NH, Niefind K, Müller J, Riebel B, Hummel W, Schomburg D (2005) Journal of Molecular Biology 349: 801-813.

Valadez-Blanco R, Livingston AG (2009) Biochemical Engineering Journal 46: 44-53.

Walsh C (1980) Accounts of Chemical Research 13: $148-155$.

Willaert R, Baron G. (1996) Reviews in Chemical Engineering 12: 5-205.

Wu W, Sidhoum M, and DeLancey GB (1994) Biotechnology and Bioengineering 44: 1217-1227.

Yamada Y, Maeda K, Mikata K (1994) Bioscience, Biotechnology, and Biochemistry 58: 1245-1257. 